\title{
A CMOS Broadband Distributed Mixer
}

\author{
Chengpeng Liu ${ }^{1, a}$, Guoqiang Wang ${ }^{1, b}$ \\ ${ }^{1}$ Sichuan Institute of Solid State Circuits China Electronics Technology Group Corp., \\ Chongqing 400060, China; \\ alcp54913@163.com, bwgq198202@yahoo.com
}

Keywords: mixer, distributed, broadband, CMOS.

\begin{abstract}
In this paper, we first analyze the structure of mixer and analyze its key parts. Secondly, it analyzes the important parameters of mixer, which is helpful to finish the design better. Finally, we pay attention to the particularity of mixer, mixer design, simulation results to meet the design requirements. This circuit has been realized by $0.18 \mu \mathrm{m}$ CMOS process. this mixer exhibits high performance.
\end{abstract}

\section{Introduction}

Modern Frequency conversion is an essential feature of the RF front-end of a wireless transceiver. The frequency conversion function is performed by a mixer or modulator, which can be considered as a special mixer. According to the circuit structure, the mixer can be divided into single-ended, single-balanced and double-balanced mixer three categories; according to whether the circuit consumes DC power, the mixer can be divided into two active and passive mixer Class; according to the frequency used by the frequency of harmonic oscillator, the mixer can be divided into fundamental and sub-harmonic mixer two categories.

Most of the early millimeter-wave mixer is implemented using diodes, mainly affected by the level of technology, the non-linear diode than the transistor, and its circuit is simple.

Gilbert multiplication unit is a double-balanced structure, with good Conversion gain and port isolation performance, this mixer is widely used in wireless transceivers. Because the classic Gilbert mixer is a three-layer transistor stack structure, so the power supply voltage is high, not suitable for low-node process, even if it is difficult to achieve low power consumption, and its noise figure is generally high. In addition, Gilbert mixer RF and LO signal input are directly connected to the transistor gate, IF is from the three-tier stacked structure of the transistor drain leads to Gilbert mixer input and output resistance are high Resistance, it is difficult to achieve bandwidth matching. To achieve higher gain and wide bandwidth performance, must be at the expense of chip area and power consumption at the expense of. In recent years, some research institutions have proposed improved Gilbert unit, namely in the trans-conductance level and switching stage to introduce high impedance transmission line to improve the working bandwidth, and the use of input instead of the transconductance balun to reduce power supply voltage requirements ${ }^{[1]}$.

The mixer described above is the fundamental mixer, in recent years as the circuit tends to a higher frequency, sub-harmonic mixing technology has become a research hotspot. The sub-harmonic mixer uses local oscillator frequency is $1 / 2,1 / 3,1 / 4$ or even less of the fundamental mixer, can effectively reduce the impact of local oscillator leakage, and has better noise Performance, but because it is high-frequency harmonic mixing, under normal circumstances the conversion gain is low, and the local oscillator signal power requirements ${ }^{[1]}$.

\section{Circuit Design Mixer Key Indicators}

With Mixer is a non-linear or time-varying components to achieve the frequency conversion function of the circuit. Usually mixer has two input ports and an output port, the symbol and function diagram shown in Fig. 1. 


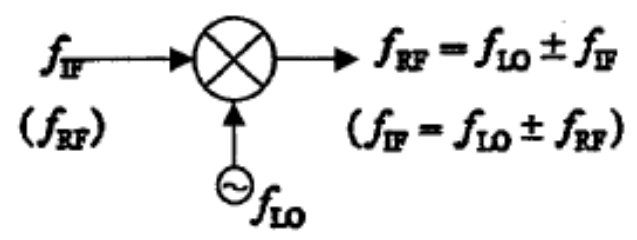

Fig.1 Mixer Functional Block Diagram

Mixer key indicators include the conversion gain, noise Fig., linearity and isolation. In the mixer design, these indicators are mutually influential, and there is a compromise between the indicators. In order to optimize the performance of mixer, we must first analyze the relationship between these indicators, through innovative design to break the trade-off between them to achieve high performance mixer ${ }^{[4 \sim 6]}$.

(1) Conversion Gain

The mixer conversion gain is defined as the ratio of the voltage conversion gain to the power conversion gain defined as the ratio of the IF voltage (power) to the RF side supply voltage (power). When the mixer input and output impedance are matched, the power gain is equal to the voltage gain, otherwise the power gain is equal to the square of the voltage gain divided by the ratio of the output to the input impedance. Since passive mixers have no gain, the usual conversion loss concept, the inverse of the conversion gain, is the noise Fig. when both input and output impedances are matched.

(2) Noise Figure

The mixer noise figure is defined as the ratio of the signal-to-noise ratio at the RF side to the signal-to-noise ratio at the IF side. For the receiver, when the mixer output IF frequency is not zero, in fact there are two different frequencies of the input signal and the local oscillator signal to produce a same IF signal. The input signals of the two different frequencies are the desired RF signal and the mirror IMG signal or the USB signal and the LSB signal, respectively, They take the local oscillator as the center of each other image signal, separated by two intermediate frequency. As the mixer will be the RF signal and the image signal is converted to IF, so not only the RF frequency noise will move to the intermediate frequency, and the image frequency position of the noise will be transferred to the intermediate frequency. If there is an image filter before the mixer, the noise figure of the mixer is SSB NF, otherwise DSB NF, which is about $3 \mathrm{~dB}$ higher than the DSB NF due to the presence of image noise in SSB mixing. Similarly for the transmitter, the SSB signal power is also less than half the DSB signal because the SSB signal has less than half the spectrum of the double-sideband signal, so the SSB NF of the mixer is about 3dB higher than the DSB NF.

(3) Linearity

Since the mixer is based on non-linear component of the work, it will produce a lot of inter-modulation products, in which the third-order inter-modulation component IM3 has the greatest impact on linearity. the linearity of the mixer can be $1 \mathrm{~dB}$ compression point or IP3 to measure.

(4) Isolation

Isolation is used to measure the degree of interaction between the various ports of the mixer, including LO to RF, LO to IF and RF to IF to the RF side. ) Isolation. In the ideal case, the local oscillator and the other two ports are completely isolated, but due to the mixer internal parasitic paths, impedance mismatch and feedback and other factors, the local oscillator signal may be affected to the RF side and the local oscillator Self-mixing, blocking the channel, so that the receiver sensitivity degradation. In addition, the local oscillator leakage signal to the RF side may also be affected by antenna radiation to other users. The local oscillator signal leakage to the IF side will cause the receiving channel signal blocking, and will deteriorate the overall receiver noise figure.

\section{Simulation Results}

Typical mixers structure typically include: (1)Single-ended mixer; (2)Single - balanced mixer; (3)Double - balanced mixer; (4)Sub - sampling mixer; (5)Distributed mixer; (6)Subharmonic mixer.

Single-ended mixer and single-balanced mixer structure is simple, low power consumption but the isolation is poor, mainly used in discrete components to achieve the mixer, while the chip is less 
used. Double-balanced mixer has the advantage of high isolation, the Gilbert mixer is a common active double-balanced mixer, the mixer has a good conversion gain, but in order to maintain good gain performance, The circuit also needs a large enough DC power consumption, its bandwidth is mainly limited by the input impedance matching, because the local oscillator and RF input are directly connected to the gate of the transistor, it is difficult to obtain a wide bandwidth, and its linearity Subject to transconductance linearity. In addition, the Gilbert mixer is a multi-layer transistor stack design, so the voltage net space is small, difficult to adapt to low-voltage low-power design. Passive double-balanced mixer is a high linearity and zero DC power consumption, but the conversion loss is generally larger, but also need a larger local oscillator power. Although the sub-sampling mixer can obtain high linearity, but its noise coefficient is very big, its so-called high linearity can be offset by its bad noise characteristic, so the dynamic range of this kind of mixer is generally smaller. In addition, sub-sampling mixer on the sampling circuit is very demanding, it is generally only suitable for low-frequency mixer circuit. Subharmonic mixer although can obtain good port isolation, but its working bandwidth depends on its matching circuit, and its conversion gain is generally low. Although the distributed mixer can obtain wide bandwidth, the better conversion gain of the active distributed mixer also requires large DC power consumption, while the passive distributed mixer is a single-tube structure, Port isolation is low and conversion loss is also high, easily lead to signal blocking and noise figure. of the deterioration.

Distributed mixer is the use of the parasitic capacitance of the transistor and the parasitic inductance of the connection cutoff frequency is very high artificial transmission line, which can achieve a wide match bandwidth. With low noise, high bandwidth and high dynamic range of performance characteristics.

Distributed mixer can be divided into active and passive forms, in which active distributed can get a certain gain but power consumption, passive distributed although no power, but the conversion loss is larger, isolation Poor, but also on the vibration power. the main disadvantage is that the chip area is large, and the circuit efficiency is low in the same gain of the power consumption compared to the other Type mixer power consumption is much higher.

In this paper, the main research for the high-frequency wideband mixer based on CMOS technology, so the use of distributed technology design and implementation of mixer broadband characteristics.

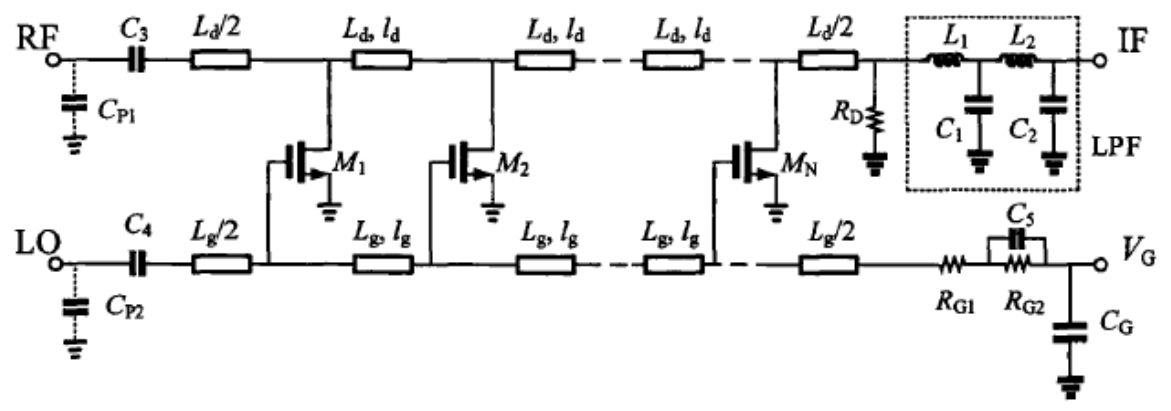

Fig.2 Broadband Distributed Mixer

This circuit has been realized by $0.18 \mu \mathrm{m}$ CMOS process. this broadband distributed mixer exhibits high performance. The circuit of the mixer is shown in Fig.2. The simulation of our mixer have been presented based on the Cadence spectre. over 5 40GHz the proposed mixer Conversion gain of mixer is more than -6dB (Fig.3), RF returen of mixer is lower than 12dB (Fig.4), LO to RF isolation is more than $20 \mathrm{~dB}$, LO to IF isolation is more than $26 \mathrm{~dB}$ (Fig.5) , Noise figure of mixer is less than 12dB(Fig.6).

Fig. 3 to Fig.6 is broadband distributed Mixer simulation curve. From the simulation curve can be seen, mixer to meet the requirements of broadband supply. 


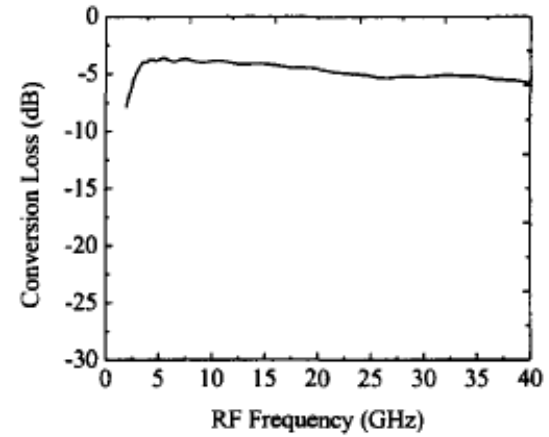

Fig.3 Conversion gain of mixer

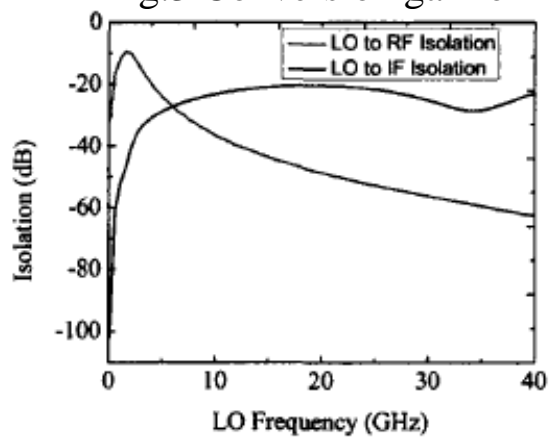

Fig.5 Isolation of mixer

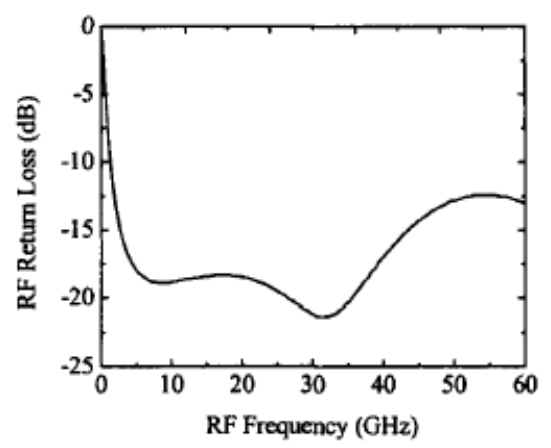

Fig.4 RF returen of mixer

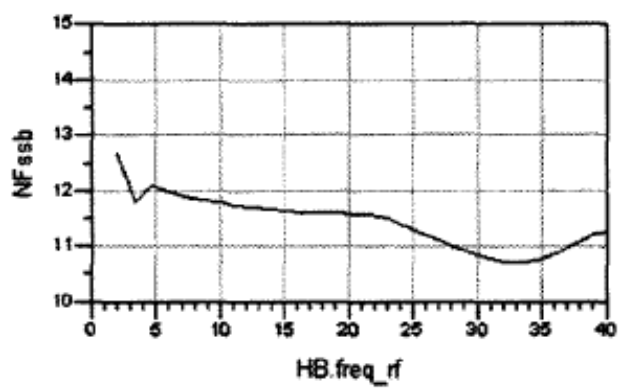

Fig.6 Noise figure of mixer

\section{Conclusion}

In this paper, we first analyze the structure of mixer and analyze its key parts. Secondly, it analyzes the important parameters of mixer, which is helpful to finish the design better. Finally, we pay attention to the particularity of mixer, mixer design, simulation results to meet the design requirements.

\section{References}

[1] Can K San dalc, Sayfe Kiaei. Analysis of Adaptive CMOS Down Conversion Mixers[C]. S-VLSI,118-121.

[2] $\mathrm{R}$ van Langevelde, et al. RF-Distortion in Deep-Submicron CMOS Technologies[J]. IEEE IEDM,807-810.

[3] Theerachet Soorapanth, Thomas H Lee. RF Linearity of Short-Channel MOSFETs[C]. Proceedings of 1St international workshop on Design of mixed-mode integrated circuits and applications, Cancun, Mexico, 1997, 81-84.

[4] P Litmanen, et al. A 2.OGHz Submicron CMOS LNA and a Downconversion Mixer[C]. IEEE ISCAS,1998, 357-359.

[5] Tsutomu Wakimoto, et al. Sub 1-V 5-GHz Band Up- and Down-Conversion Mixer Cores in 0.35um CMOS[C]. IEEE Symposium o VLSI Circuits Digest of Technical Papers, 2000, 98-99.

[6] Ertan Zencir, Numan S Dogan, Ercument Arvas. A Low-power CMOS Mixer for Low-IFReceivers[C]. IEEE RAWCON, 2002, 157-160. 